OPEN ACCESS

Edited by:

Yuming Jiang,

Stanford University, United States

Reviewed by:

Xiaohu Li,

First Affiliated Hospital of Anhui

Medical University, China

Mathieu Hatt,

Institut National de la Santé et de la

Recherche Médicale (INSERM),

France

${ }^{*}$ Correspondence:

Zixing Huang

zixinghuang@hotmail.com

Jiankun Hu

hujkwch@126.com

${ }^{+}$These authors have contributed equally to this work and share

first authorship

Specialty section: This article was submitted to

Cancer Imaging and

Image-directed Interventions,

a section of the journal

Frontiers in Oncology

Received: 15 September 2021 Accepted: 09 November 2021 Published: 03 December 2021

Citation:

Liu D, Zhang W, Hu F, Yu P, Zhang $X$, Yin $H$, Yang L, Fang $X$, Song $B, W u B$, Hu J and Huang Z (2021) A Bounding Box-Based Radiomics Model for

Detecting Occult Peritoneal

Metastasis in Advanced Gastric Cancer: A Multicenter Study.

Front. Oncol. 11:777760.

doi: 10.3389/fonc. 2021.777760

\section{A Bounding Box-Based Radiomics Model for Detecting Occult Peritoneal Metastasis in Advanced Gastric Cancer: A Multicenter Study}

\author{
Dan Liu ${ }^{1 \dagger}$, Weihan Zhang ${ }^{2 t}$, Fubi Hu ${ }^{3 \dagger}$, Pengxin Yu ${ }^{4}$, Xiao Zhang ${ }^{5}$, Hongkun Yin ${ }^{4}$, \\ Lanqing Yang ${ }^{1}$, Xin Fang ${ }^{1}$, Bin Song ${ }^{1}$, Bing $\mathrm{Wu}^{1}{ }^{1}$, Jiankun $\mathrm{Hu}^{2 *}$ and Zixing Huang ${ }^{1 *}$ \\ ${ }^{1}$ Department of Radiology, West China Hospital, Sichuan University, Chengdu, China, ${ }^{2}$ Department of Gastrointestinal \\ Surgery and Laboratory of Gastric Cancer, Collaborative Innovation Center for Biotherapy, State Key Laboratory of \\ Biotherapy, West China Hospital, Sichuan University, Chengdu, China, ${ }^{3}$ Department of Radiology, First Affiliated Hospital of \\ Chengdu Medical College, Chengdu, China, ${ }^{4}$ Institute of Advanced Research, Infervision, Beijing, China, ${ }^{5}$ Department of \\ Radiology, People's Hospital of Leshan, Leshan, China
}

Purpose: To develop a bounding box (BBOX)-based radiomics model for the preoperative diagnosis of occult peritoneal metastasis (OPM) in advanced gastric cancer (AGC) patients.

Materials and Methods: 599 AGC patients from 3 centers were retrospectively enrolled and were divided into training, validation, and testing cohorts. The minimum circumscribed rectangle of the ROls for the largest tumor area (R_BBOX), the nonoverlapping area between the tumor and R_BBOX (peritumoral area; PERI) and the smallest rectangle that could completely contain the tumor determined by a radiologist (M_BBOX) were used as inputs to extract radiomic features. Multivariate logistic regression was used to construct a radiomics model to estimate the preoperative probability of OPM in AGC patients.

Results: The M_BBOX model was not significantly different from R_BBOX in the validation cohort [AUC: M_BBOX model $0.871(95 \% \mathrm{Cl}, 0.814-0.940)$ vs. R_BBOX model 0.873 (95\% Cl, 0.820-0.940); $p=0.937]$. M_BBOX was selected as the final radiomics model because of its extremely low annotation cost and superior OPM discrimination performance (sensitivity of $85.7 \%$ and specificity of $82.8 \%$ ) over the clinical model, and this radiomics model showed comparable diagnostic efficacy in the testing cohort.

Conclusions: The BBOX-based radiomics could serve as a simpler reliable and powerful tool for the preoperative diagnosis of OPM in AGC patients. And M_BBOX-based radiomics is simpler and less time consuming.

Keywords: gastric cancer, peritoneal metastasis, radiomics, bounding box, computed tomography 


\section{INTRODUCTION}

Gastric cancer (GC) is one of the most common and deadly carcinomas in the world (1). Peritoneal metastasis (PM), one of the most common forms of metastasis in GC, occurs in 53\%$80 \%$ of GC patients with distant metastasis $(2,3)$ and is generally regarded as an incurable condition with a poor prognosis $(4,5)$. It was reported that the median survival time among PM patients is 3-6 months (5), and the treatment options are limited (6). New treatment strategies such as intraperitoneal chemotherapy and extensive intraoperative peritoneal lavage are associated with an improved prognosis for those patients (7-9). Therefore, noninvasive preoperative detection of PM of advanced gastric cancer (AGC) is crucial for avoiding unnecessary surgery and selecting optimal therapy in clinical practice.

CT is recommended as the first-line imaging modality for the detection of PM (10). However, PMs $(<5 \mathrm{~mm})$ are frequently missed on CT images (11). In 10 to $30 \%$ of patients with negative CT images, intraperitoneal metastases are found during either laparoscopy or surgical exploration $(11,12)$, called occult peritoneal metastases (OPMs).

Artificial intelligence, especially deep learning, has been explored in predicting PM in GC $(13,14)$, such as previous research from our team (14). However, the interpretation is under elucidated due to its nature of "black box". Radiomics is an emerging field focusing on the high-dimensional mineable feature set captured from imaging data using a series of quantitative characteristic algorithms. The effective extraction and modeling of digital information is expected to aid in the assessment and differential diagnosis of gastric tumors (15-20). For OPMs in AGC, some studies (21-23) have utilized radiomics analysis of preoperative CT texture features to make a preoperative, noninvasive OPM diagnosis. All regions of interest (ROIs) were drawn manually in these studies. However, the manual annotation of many medical images is time consuming, expensive, and inefficient. In addition, most medical image annotations require a certain level of expertise, which can add additional work for the physician. Recently, we applied another annotation method-an easy-to-use, timesaving and inexpensive method referred to as the bounding box (BBOX) - that has been used in some clinical areas (24-28), such as segmentation, diagnosis, and classification, but has not yet been applied to the radiomics analysis of OPM in GC patients.

The BBOX, which contains both the tumor tissue and peritumoral area (PERI), covers more regions than manual annotation of the tumor alone. A few studies have revealed that the combined radiomic signature of intratumoral and peritumoral regions can provide valuable information for the prediction of the Lauren classification of GC (29), the pathological complete response to neoadjuvant chemoradiation of esophageal squamous cell carcinoma (30), and the outcomes and chemotherapy response in GC $(31,32)$.

Therefore, in this study, we hypothesized that ROI annotation by BBOX might provide adequate information for the diagnosis of OPM. We aimed to develop and validate a BBOX-based radiomics model for the preoperative, noninvasive diagnosis of OPM in AGC patients.

\section{MATERIALS AND METHODS}

The radiomics processing flowchart of this study is shown in Figure 1.

\section{Patients}

This multicenter retrospective study was approved by the institutional review board of each center, and the informed consent requirement was waived. A total of 599 patients from three centers were divided into three cohorts: one training cohort (395 patients from center 1), one internal validation cohort (149 patients from center 1), and one external testing cohort (55 patients from center 2 and center 3). The details of PM status confirmation are described in Section $\mathbf{1}$ in the Supplementary Material. The inclusion criteria and exclusion criteria are available in Section 2 in the Supplementary Material.

\section{CT Image Acquisition and Data Preparation}

The details of the CT protocol are presented in Section 3 and Table S1 in the Supplementary Material. Portal vein phase CT images were first exported to ITK-SNAP software (version 2.2.0; www.itksnap.org) for manual segmentation. GC lesions were then manually annotated by a radiologist with 5 years of experience in gastroenterology imaging and confirmed by another abdominal specialist with 14 years of experience.

These two radiologists reviewed all slices obtained from each patient, selected the slice with the largest tumor area, and manually delineated the lesion to obtain the final ROIs. The $\mathrm{BBOX}$ was obtained by calculating the minimum circumscribed rectangle of the ROI, as shown in Figure 2A, called the R_BBOX. The nonoverlapping area between the ROI and BBOX was regarded as the PERI. For all patients, the radiologist was required to determine the smallest rectangle that could completely contain the tumor area and directly perform BBOX annotation. The manually obtained BBOX annotation was called M_BBOX. An example is shown in Figure 2B, as indicated by the blue line.

\section{Radiomic Feature Extraction}

Radiomic features were extracted using Pyradiomics (33), an open-source radiomics toolbox. Eight classes of stable radiomic features were extracted according to the standards in the Image Biomarker Standardization Initiative (34). Ultimately, 119 quantitative two-dimensional (2D) radiomics features were extracted for each annotation of the ROI, R_BBOX, PERI and M_BBOX. For each patient in the training cohort and validation cohort, we constructed an annotation type called MERGE, which includes the features of both the ROI and the PERI. Thus, the number of features in the MERGE region was 238. The MERGE approach is another way to analyze the tumor area and PERI at the same time. 


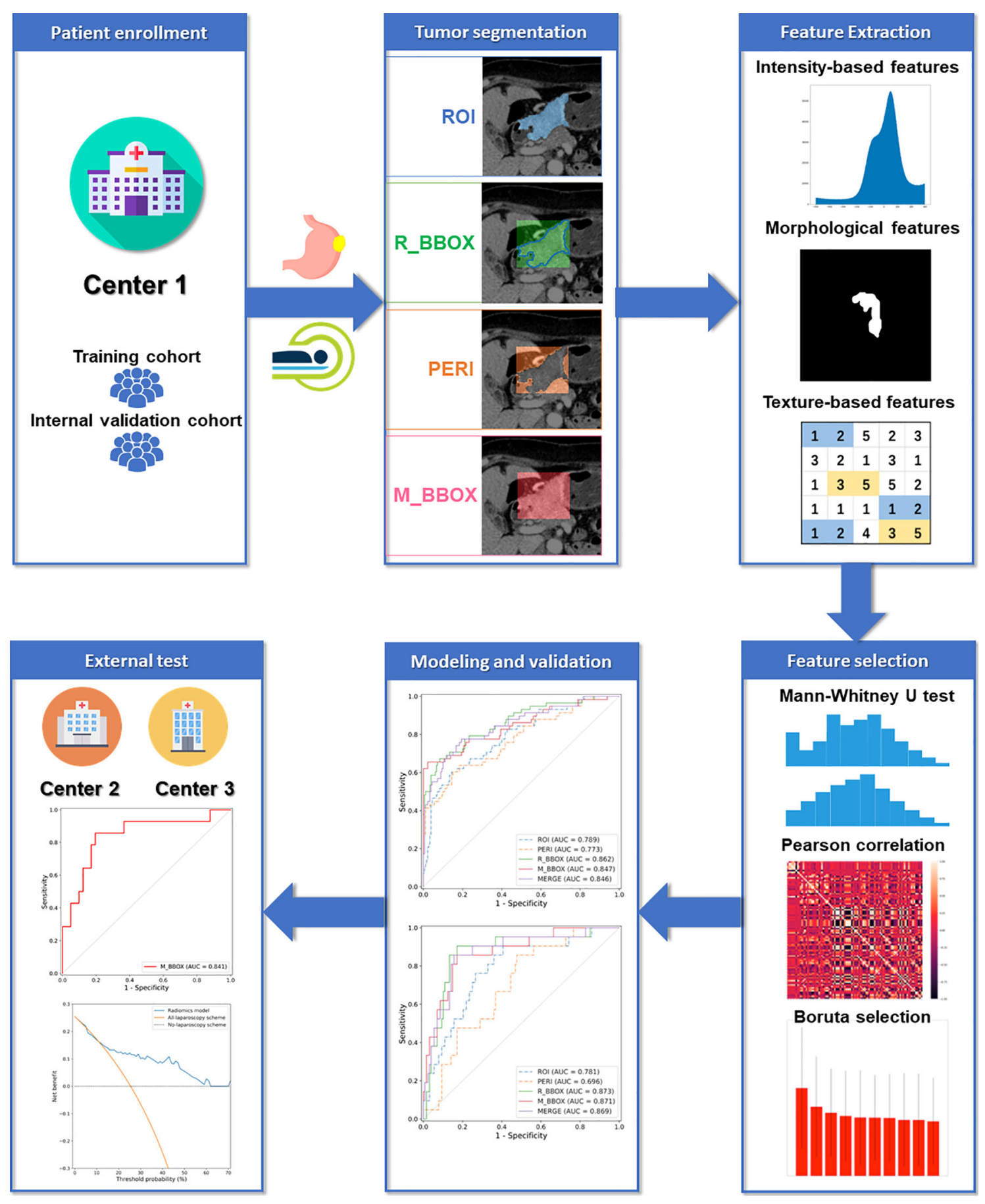

FIGURE 1 | Radiomics processing flowchart.

\section{Feature Selection in Terms of Reproducibility}

Two months after data annotation, 50 patients were randomly selected from the training cohort and reannotated by the previous radiologist. The same annotation rules were applied; that is, the ROIs and M_BBOXes of 50 patients were manually delineated. The R_BBOX and PERI for each patient could be obtained based on the ROI. For each patient, the radiomics features of the ROI, R_BBOX, PERI, M_BBOX and MERGE could be obtained. To evaluate the intraobserver agreement between the two repeated annotations, intraclass correlation coefficients (ICCs) were utilized. An ICC greater than 0.75 was considered a sign of reproducibility for the observer, and features 

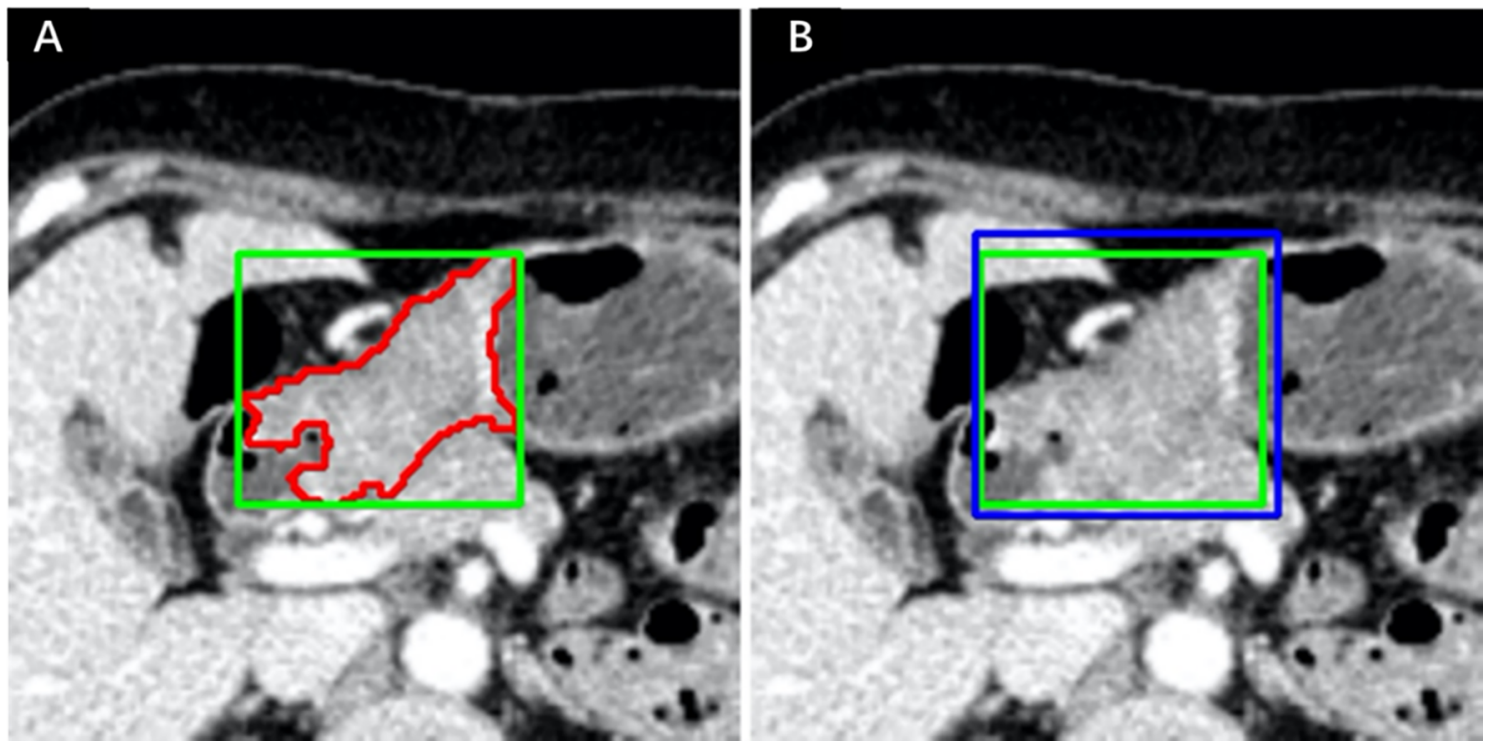

FIGURE 2 | Annotation example in one patient. (A) ROI and R_BBOX indicated by the red and green lines, respectively. (B) M_BBOX indicated by the blue line; R_BBOX same as in (A).

with ICCs lower than 0.75 were excluded in the following feature selection process.

\section{Feature Selection and Classifier Modeling}

As shown in Section 4 in the Supplement, the feature selection process included three steps, with the aim of avoiding overfitting during the model-building process and potential biases associated with the results.

After the three-step feature selection process, the remaining radiomics features were used to construct a multivariate logistic regression model for OPM positivity prediction, namely, the radiomics model.

We used independent predictors of preoperative clinical characteristics to build a clinical model by multivariate logistic regression for comparison. The independent predictors were also screened by multivariate logistic regression analysis. Backward stepwise selection was performed based on the Akaike information criterion (AIC) (35).

\section{Four Sizes of Input Boxes}

Four sizes of input boxes containing the primary tumor with different amounts of surrounding tissues were used to evaluate their diagnostic performance, as shown in Section 6 in the Supplement.

\section{Statistical Analysis}

Differences in continuous variables were analyzed with the Mann-Whitney $U$ test, and differences in categorical variables were analyzed with the chi-squared test. The radiomic classification model and the clinical classification model were evaluated with the validation cohort. The variance in performance was assessed using receiver operating characteristic (ROC) curve analysis and quantified by assessing the areas under the ROC curves (AUCs) (36). The DeLong test $(37,38)$ was used for statistical comparisons of ROC curves. Considering the class imbalance in the validation cohort and testing cohort, bootstrapping $(\mathrm{n}=1000)$ was used to calculate tfrhe 95\% confidence interval (CI). A decision curve was plotted to evaluate model efficacy by quantifying the net benefits at different probability thresholds. All statistical analyses were performed with R software (version 3.5.0; http:// www.Rproject.org) and SPSS 22.0 (IBM, Armonk, NY, USA). Differences with a two-tailed p-value less than 0.05 were considered statistically significant.

\section{RESULTS}

\section{Clinical Characteristics}

The demographic information is shown in Table 1. Table 1 shows significant differences between the lesion location and Borrmann type between OPM-positive and OPM-negative patients in the training cohort $(\mathrm{p}<0.001)$. There were no significant differences in age, sex, carcinoembryonic antigen (CEA), or carbohydrate antigen 19-9 (CA19-9) between OPM-positive and OPM-negative patients in the entire cohort.

\section{Clinical Model}

Multivariate logistic regression analysis identified location$\mathrm{L} / \mathrm{L}+\mathrm{D}[\beta=-0.791, \mathrm{OR}=0.453$ (95\% CI, 0.247-0.832), $P=0.010]$ and Borrmann type $[\beta=-1.132$, OR $=3.103$ (95\% CI, 1.504-6.401), $P=0.002]$ as independent predictors of OPM status. A clinical model incorporating the location- $\mathrm{L} / \mathrm{L}+\mathrm{D}$ and the Borrmann type was developed. 
TABLE 1 | Characteristics of the patients.

\begin{tabular}{|c|c|c|c|c|c|c|c|c|c|}
\hline \multirow[t]{2}{*}{ Characteristic } & \multicolumn{2}{|c|}{ Training cohort $(n=395)$} & \multirow[b]{2}{*}{$p$} & \multicolumn{2}{|c|}{ Validation cohort $(n=149)$} & \multirow[b]{2}{*}{$p$} & \multicolumn{2}{|c|}{ Testing cohort $(n=55)$} & \multirow[b]{2}{*}{$p$} \\
\hline & $\begin{array}{l}\text { OPM-Pos } \\
(n=58)\end{array}$ & $\begin{array}{l}\text { OPM-Neg } \\
(n=337)\end{array}$ & & $\begin{array}{l}\text { OPM-Pos } \\
(n=21)\end{array}$ & $\begin{array}{l}\text { OPM-Neg } \\
(n=128)\end{array}$ & & $\begin{array}{l}\text { OPM-Pos } \\
(n=14)\end{array}$ & $\begin{array}{c}\text { OPM-Neg } \\
(n=41)\end{array}$ & \\
\hline Age (years, mean \pm SD) & $58.17 \pm 13.24$ & $57.99 \pm 11.74$ & 0.451 & $55.86 \pm 16.33$ & $60.41 \pm 10.76$ & 0.121 & $60.61 \pm 10.71$ & $66.57 \pm 12.34$ & 0.122 \\
\hline $\operatorname{Sex}(N, \%)$ & & & 0.270 & & & 0.469 & & & \\
\hline Male & $34(58.6 \%)$ & $226(67.1 \%)$ & & $12(57.1 \%)$ & 87 (68.0\%) & & $11(78.6 \%)$ & $26(63.4 \%)$ & \\
\hline Female & $24(41.4 \%)$ & $111(32.9 \%)$ & & 9 (42.9\%) & 41 (32.0\%) & & 3 (21.4\%) & $15(36.6 \%)$ & \\
\hline Location (N, \%) & & & 0.002 & & & 0.116 & & & 0.917 \\
\hline $\mathrm{U} / \mathrm{U}+\mathrm{M}$ & 12 (20.7\%) & $68(20.2 \%)$ & & 3 (14.3\%) & $36(28.1 \%)$ & & $2(14.3 \%)$ & 9 (22.0\%) & \\
\hline $\mathrm{M} / \mathrm{M}+\mathrm{L}$ & $20(34.5 \%)$ & 63 (18.7\%) & & $6(28.6 \%)$ & $18(14.1 \%)$ & & $3(21.4 \%)$ & $8(19.5 \%)$ & \\
\hline $\mathrm{L} / \mathrm{L}+\mathrm{D}$ & 18 (31.0\%) & $181(53.7 \%)$ & & 9 (42.9\%) & 63 (49.2\%) & & $8(57.1 \%)$ & $19(46.3 \%)$ & \\
\hline U+E & $1(1.7 \%)$ & $11(3.3 \%)$ & & $0(0.0 \%)$ & 5 (3.9\%) & & $0(0.0 \%)$ & $3(7.3 \%)$ & \\
\hline Whole stomach & 7 (12.1\%) & $14(4.2 \%)$ & & $3(14.3 \%)$ & $6(4.7 \%)$ & & $1(7.1 \%)$ & $2(4.9 \%)$ & \\
\hline Borrmann type (N, \%) & & & 0.000 & & & 0.009 & & & 0.123 \\
\hline Types 1,2 & $48(82.8 \%)$ & $194(57.6 \%)$ & & $18(85.7 \%)$ & 71 (55.5\%) & & $1(10.0 \%)$ & 9 (39.1\%) & \\
\hline Types 3, 4 & 10 (17.2\%) & $143(42.4 \%)$ & & $3(14.3 \%)$ & 57 (44.5\%) & & 9 (90.0\%) & 14 (60.9\%) & \\
\hline Unknown & 0 & 0 & & 0 & 0 & & 4 & 18 & \\
\hline CEA (N, \%) & & & 0.313 & & & 0.128 & & & 0.616 \\
\hline Normal & $36(62.1 \%)$ & 235 (69.7\%) & & $18(85.7 \%)$ & 88 (68.8\%) & & $8(80.0 \%)$ & 28 (87.5\%) & \\
\hline Elevated & 22 (37.9\%) & 102 (30.3\%) & & $3(14.3 \%)$ & $40(31.2 \%)$ & & 2 (20.0\%) & $4(12.5 \%)$ & \\
\hline Unknown & 0 & 0 & & 0 & 0 & & 4 & 9 & \\
\hline CA19-9 (N, \%) & & & 0.091 & & & 0.221 & & & 1.000 \\
\hline Normal & 37 (63.8\%) & 254 (75.4\%) & & 11 & 88 & & 8 (80.0\%) & $26(81.3 \%)$ & \\
\hline Elevated & 21 (36.2\%) & 83 (24.6\%) & & 10 & 40 & & $2(20.0 \%)$ & $6(18.7 \%)$ & \\
\hline Unknown & 0 & 0 & & 0 & 0 & & 4 & 9 & \\
\hline
\end{tabular}

\section{Annotation Type Analysis}

We evaluated the impact of different annotation types on the performance of the radiomics model in the validation cohort. As shown in Table 2, after the three-step feature selection process, the number of radiomics features for the different annotation types was $3,3,2,3$ and 6 . The radiomics model based on R_BBOX yielded an AUC of 0.873 (95\% CI, 0.820-0.940), which was significantly better than the ROI model [AUC: 0.781 (95\% CI, 0.710-0.863); $p=0.047$. Similarly, in the radiomics model that combined the tumor area and the PERI for analysis, an improvement in the predictive performance was noted [AUC: M_BBOX 0.871 (95\% CI, 0.814-0.940), MERGE 0.869 (95\% CI, 0.811-0.938)], although there was no significant difference by the Delong test ( $p$ : M_BBOX 0.081, MERGE 0.080). On the other hand, for the radiomics model that used only PERI for analysis, a decrease in the predictive performance was observed, with an AUC of 0.696 (95\% CI, 0.620-0.785). After setting the threshold, the specificity of each model was compared when the sensitivity reached 0.8 . The $\mathrm{R} \_$BBOX radiomics model produced the highest specificity, and the specificity of all radiomics models that combined tumor area and PERI exceeded 0.85 (specificity: R_BBOX 0.867 , M_BBOX 0.852 , MERGE 0.859). In contrast, the radiomics models using only tumor area or PERI had lower specificity (specificity: ROI 0.672 , PERI 0.531). The ROC curves of the different radiomics models are shown in Figure 3.

M_BBOX was used as the final radiomics model because of its high prediction accuracy and extremely low annotation cost. The prediction score of the M_BBOX radiomics model was calculated as follows: $-1.8863+(-0.3683 *$ GLRLM_RunLengthNon UniformityNormalized $)+(-0.0416$ * GLDM_GrayLevel NonUniformity $)+(-0.5014$ * Shape2D_PerimeterSurfaceRatio $)$.

\section{Comparison of the Clinical Model With the Radiomics Model}

The comparison of the discrimination performance of the radiomics model and the clinical model in the validation cohort is shown in Section $\mathbf{5}$ and Table S2 in the Supplementary

TABLE 2 | Performance of the radiomics models with different annotation types.

\begin{tabular}{lccccc}
\hline Annotation type & Feature count & AUC (95\% Cl) & Sensitivity & Specificity \\
\hline ROI & 3 & $0.781(0.710-0.863)$ & 0.810 & 0.672 \\
PERI & 3 & $0.696(0.620-0.785)$ & 0.810 & 0.047 \\
R_BBOX & 2 & $0.873(0.820-0.940)$ & 0.810 & 0.158 & 0.867 \\
M_BBOX & 3 & $0.871(0.814-0.940)$ & 0.810 & 0.852 \\
MERGE & 6 & $0.869(0.811-0.938)$ & 0.810 & 0.047 & 0.859 \\
\hline
\end{tabular}

$p$ is the Delong test result for ROIs versus other annotation types.

$p^{*}$ is the Delong test result for BBOX versus other annotation types.

$R O I$, region of interest; PERI, peritumoral area; $R \_B B O X$, bounding box was obtained by calculating the minimum circumscribed rectangle of the ROI; $M \_B B O X$, manual bounding box; AUC, area under the curve; $\mathrm{Cl}$, confidence interval. 


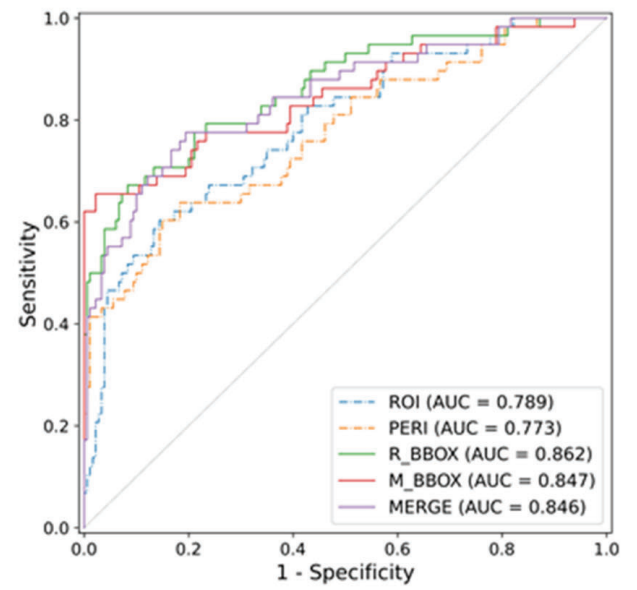

C

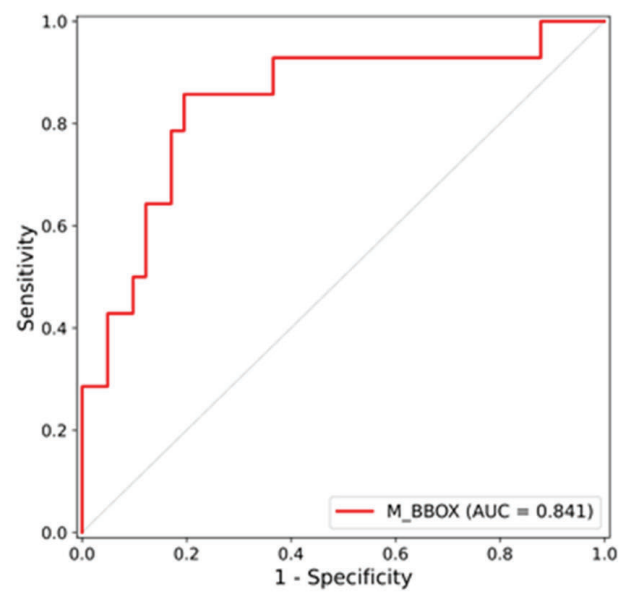

B

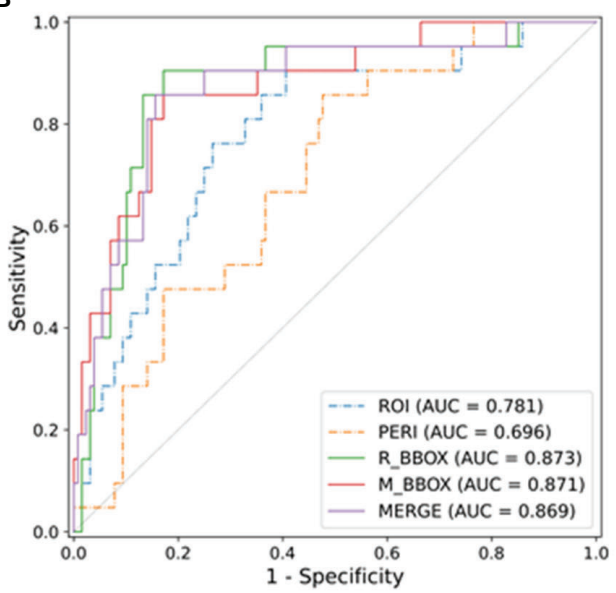

FIGURE 3 | ROC curves of the radiomics models in the training cohort (A), validation cohort (B) and external test cohort (C), respectively.

Material. The DeLong test showed that the diagnostic performance of the radiomics model was significantly better than that of the clinical model $(p=0.007)$. Both the sensitivity and specificity values of the radiomics model were higher than those of the clinical model (sensitivity: 0.857 vs. 0.524 ; specificity: 0.828 vs. 0.719).

As shown in Section $\mathbf{5}$ and Table $\mathbf{S 3}$ in the Supplementary Material, in the testing cohort, the AUC, sensitivity and specificity values of the radiomics model for all patients were 0.841 (95\% CI, 0.697-0.956), 0.857 and 0.805, respectively. Considering that the clinical characteristics of some patients in the testing cohort were incomplete, a subset of the testing cohort containing 24 patients with complete clinical information was constructed. The predictive performances in the testing cohort (subset) are described in Section 5 and Table S3 in the Supplementary Material. Although there was no significant difference by the Delong test, the AUC of the radiomics model was better than that of the clinical model (AUC: radiomics model 0.889 , clinical model 0.648 ), and it had higher specificity under the same sensitivity (specificity: radiomics model 0.778 , clinical model 0.333).

\section{Clinical Use}

Decision curve analysis (DCA) was used to evaluate the benefits of the radiomics model and both the all-laparoscopy and nolaparoscopy schemes. The net benefit of the no-laparoscopy scheme was always 0 . The all-laparoscopy scheme means that additional laparoscopy was performed on all patients. The DCA results for the validation cohort are shown in Figure S2A. The radiomics model had the highest net benefit when the threshold probability in clinical decision-making was between 5\% and $45 \%$. Figure S2B shows the DCA results for the testing cohort, which indicated that the radiomics model had the highest net benefit when the threshold probability was between $10 \%$ and $60 \%$.

\section{Four Sizes of Input Boxes}

Our results indicated that the model achieved the highest AUC by using BBOX as input. (Figures S2, S3). However, when the 
size of the BBOX increased by $10 \mathrm{~mm}, 15 \mathrm{~mm}$, or no limit, the performance of the models worsened, which might be because the larger the BBOX is, the more tissues are covered.

\section{DISCUSSION}

In this multicenter study, we developed a radiomics model based on 2D images annotated according to the BBOX containing the primary tumor and PERI to identify OPM in AGC patients prior to surgical treatment. The BBOX radiomics model had high diagnostic performance in the validation cohort, testing cohort (all), and testing cohort (subset).

The BBOX, an easy-to-use tool, can reduce the need for additional labeling and has been used in many clinical investigations (24-28). The region of the BBOX containing both the primary tumor and nearby peritoneum is larger than the ROI of the primary tumor. Recent reports $(26,29-32,39,40)$ have illustrated that peritumoral tissue-based radiomics analysis may reveal valuable information for diagnosis, prognosis and treatment response evaluations. Therefore, in our study, we focused on both the characteristic features of the primary tumor and the peritumoral tissue delineated with the BBOX to develop a radiomics model for the noninvasive diagnosis of OPM.

The analysis of peritumoral tissues surrounding the tumor mass can reveal important information related to tumor aggressiveness; it can reflect lymphovascular invasion, lymphangiogenesis, and angiogenesis (41-43) and provide other information that can be used for diagnostic and prognostic predictions (26, 29-32, 39-42). Moreover, such information may be effectively captured by radiomics analysis $(26,29-32,39,40)$. In our study, the nonoverlapping area between the tumor and R_BBOX was regarded as the PERI, which is theoretically a part of the whole peritumoral region. Our results indicated that the predictive value of PM based on the peritumoral region alone was limited, worse than that of the primary tumor alone, but showed significant improvement when integrated with the ROI of the primary tumor. This may be because the primary tumor, the main disease component, theoretically holds more information about tumor phenotypes than that of PERI, and BBOX covers more regions than the mass or peritoneal region alone.

Few studies (23-28) have investigated radiomic applications for PM diagnosis using CT. Liu et al. (21) and Kim et al. (22) focused on the preoperative CT texture features of the primary tumor area and the omentum area, respectively, to evaluate the possibility of PM in AGCs instead of considering the primary tumor and the surrounding tissue. Dong et al. (23) conducted a radiomics study using the CT phenotypes of primary tumors and the nearby peritoneum to accurately predict OPM in AGC patients. In their study, the diagnostic value of either the peritoneum or the mass was worse than the merged value for the detection of PM, which is consistent with our conclusions. However, they chose regions near the peritoneum rather than the primary tumor, which may be relatively far away from the mass. The delineated ROI of the peritoneum was part of the peritumoral tissue, and the annotated peritoneal area was $>2$ $\mathrm{cm}$ (2), which might not be representative of the entire surrounding condition of the mass on $2 \mathrm{D} C \mathrm{CT}$ images. In addition, manual sketching is a time-consuming and laborious process. The peritoneum is a wide and irregular structure without clear boundaries and forms multiple reflex structures (such as ligaments). Therefore, it is impossible to outline the whole peritoneum. This previous study also selected the local peritoneum, a small area. Although the researchers provided a rationale for selecting this method, delineating the peritoneal area of interest is still very subjective, and thus, it is difficult to determine reproducibility. We used the BBOX method to include all the information on the primary lesion at the 2D level and the information on the peritoneal area surrounding the lesion. These peritoneal areas were not selected subjectively; rather, they were selected incidentally based on their location relative to the primary lesion. Therefore, we believe that our delineation model is simple, easy to use, and reproducible.

Our results showed that by merging the ROI with the PERI, the radiomics model could achieve a higher AUC. This presented a question: what $\mathrm{BBOX}$ size would achieve satisfactory diagnostic value for identifying OPM? When the size of the BBOX increased by $10 \mathrm{~mm}, 15 \mathrm{~mm}$, or no limit, the performance of the models worsened, which might be because the larger the BBOX is, the more tissues are covered. The tissues far away from the carcinoma provided less or no information about the neoplasms. Considering the lower annotation cost and similar AUC of the M_BBOX model compared with the R_BBOX model, the M_BBOX radiomics model was ultimately selected as the final radiomics model.

Laparoscopy remains a useful procedure for evaluating PM status (44-46), but it is invasive and expensive, and its specific applications for GC remain controversial. Our results revealed that the clinical model could not accurately predict PM status. The value of the radiomics model was then assessed by DCA. If the predicted probability of OPM is between 10 and 45\%, more cases of undetected OPM on conventional CT images can be detected by the radiomics model than by the all-laparoscopy or no-laparoscopy scheme, avoiding unnecessary surgical procedures and extra costs. Furthermore, patients deemed to have a high possibility of OPM by the radiomics model may undergo diagnostic laparoscopy for further identification, helping to guide proper treatment.

\section{LIMITATIONS}

There are several limitations to this study. First, we focused on $2 \mathrm{D}$ slices of lesions rather than whole lesions. In future work, automatic segmentation of the whole tumor is worth developing to better predict OPM status. Second, our study was retrospective in nature, and some initially available clinical factors were analyzed. Third, a small number of patients from two external centers were used to evaluate the generalizability of the radiomics 
model; however, large independent patient cohorts are still needed to validate our results.

\section{CONCLUSIONS}

The radiomics model based on M_BBOX had higher diagnostic performance for the preoperative detection of OPM in AGC than the radiomics models based on tumor tissue or peritumoral tissue alone. In addition, its annotation is simpler and less time consuming.

\section{DATA AVAILABILITY STATEMENT}

The raw data supporting the conclusions of this article will be made available by the authors, without undue reservation.

\section{ETHICS STATEMENT}

The studies involving human participants were reviewed and approved by Ethics Committee on Biomedical Research, West China Hospital of Sichuan University. The patients/participants provided their written informed consent to participate in this study.

\section{AUTHOR CONTRIBUTIONS}

DL: data curation, software, formal analysis, validation, investigation, methodology, and writing original draft. WZ: data curation, formal analysis, validation, investigation,

\section{REFERENCES}

1. Bray F, Ferlay J, Soerjomataram I, Siegel RL, Torre LA, Jemal A. Global Cancer Statistics 2018: GLOBOCAN Estimates of Incidence and Mortality Worldwide for 36 Cancers in 185 Countries. CA Cancer J Clin (2018) 68:394424. doi: 10.3322/caac.21492

2. Sarela AI, Yelluri S. Gastric Adenocarcinoma With Distant Metastasis: Is Gastrectomy Necessary. Arch Surg (2007) 142:143-9; discussion 149. doi: 10.1001/archsurg.142.2.143

3. Coccolini F, Cotte E, Glehen O, Lotti M, Poiasina E, Catena F, et al. Intraperitoneal Chemotherapy in Advanced Gastric Cancer. Meta-Analysis of Randomized Trials. Eur J Surg Oncol (2014) 40:12-26. doi: 10.1016/ j.ejso.2013.10.019

4. Pasqual EM, Bertozzi S, Londero AP, Brandolin D, Mariuzzi L, De Pellegrin A, et al. Microscopic Peritoneal Carcinomatosis in Gastric Cancer: Prevalence, Prognosis and Predictive Factors. Oncol Lett (2018) 15:710-6. doi: 10.3892/ol.2017.7442

5. Thomassen I, van Gestel YR, van Ramshorst B, Luyer MD, Bosscha K, Nienhuijs SW, et al. Peritoneal Carcinomatosis of Gastric Origin: A Population-Based Study on Incidence, Survival and Risk Factors. Int J Cancer (2014) 134:622-8. doi: 10.1002/ijc.28373

6. Kitayama J, Ishigami H, Yamaguchi H, Sakuma Y, Horie H, Hosoya $\mathrm{Y}$, et al. Treatment of Patients With Peritoneal Metastases From Gastric Cancer. Ann Gastroenterol Surg (2018) 2:116-23. doi: 10.1002/ ags 3.12060 methodology, and writing original draft. $\mathrm{FH}$ : resources, data curation, software, formal analysis, validation, investigation, and writing original draft. PY: data curation, software, formal analysis, validation, investigation, methodology, and visualization. XZ: resources, data curation, software, formal analysis, and validation. HY: conceptualization, data curation, software, formal analysis, validation, and methodology. LY: data curation, formal analysis, and investigation. XF: data curation, formal analysis, and investigation. BS: resources, data curation, formal analysis, supervision, and methodology. BW: resources, data curation, formal analysis, supervision, and methodology. JH: conceptualization, resources, data curation, formal analysis, supervision, funding acquisition, validation, investigation, methodology, project administration, and writing review and editing. $\mathrm{ZH}$ : conceptualization, resources, data curation, formal analysis, supervision, validation, investigation, methodology, writing original draft, project administration, and writing review and editing. All authors contributed to the article and approved the submitted version.

\section{FUNDING}

This work was supported by the $1^{\star} 3^{\star} 5$ Project for Disciplines of Excellence, West China Hospital, Sichuan University (No. ZY2017304).

\section{SUPPLEMENTARY MATERIAL}

The Supplementary Material for this article can be found online at: https://www.frontiersin.org/articles/10.3389/fonc.2021.777760/ full\#supplementary-material

7. Misawa K, Mochizuki Y, Ohashi N, Matsui T, Nakayama H, Tsuboi K, et al. A Randomized Phase III Trial Exploring the Prognostic Value of Extensive Intraoperative Peritoneal Lavage in Addition to Standard Treatment for Resectable Advanced Gastric Cancer: CCOG 1102 Study. Jpn J Clin Oncol (2014) 44:101-3. doi: 10.1093/jjco/hyt157

8. Kim G, Chen E, Tay AY, Lee JS, Phua JN, Shabbir A, et al. Extensive Peritoneal Lavage After Curative Gastrectomy for Gastric Cancer (EXPEL): Study Protocol of an International Multicentre Randomised Controlled Trial. Jpn J Clin Oncol (2017) 47:179-84. doi: 10.1093/jjco/hyw153

9. Guo J, Xu A, Sun X, Zhao X, Xia Y, Rao H, et al. Combined Surgery and Extensive Intraoperative Peritoneal Lavage vs Surgery Alone for Treatment of Locally Advanced Gastric Cancer: The SEIPLUS Randomized Clinical Trial. JAMA Surg (2019) 154:610-6. doi: 10.1001/jamasurg.2019.0153

10. Smyth EC, Verheij M, Allum W, Cunningham D, Cervantes A, Arnold D. Gastric Cancer: ESMO Clinical Practice Guidelines for Diagnosis, Treatment and FollowUp. Ann Oncol (2016) 27:v38-38v49. doi: 10.1093/annonc/mdw350

11. Kim SJ, Kim HH, Kim YH, Hwang SH, Lee HS, Park DJ, et al. Peritoneal Metastasis: Detection With 16- or 64-Detector Row CT in Patients Undergoing Surgery for Gastric Cancer. Radiology (2009) 253:407-15. doi: 10.1148/radiol.2532082272

12. Burbidge S, Mahady K, Naik K. The Role of CT and Staging Laparoscopy in the Staging of Gastric Cancer. Clin Radiol (2013) 68:251-5. doi: 10.1016/ j.crad.2012.07.015

13. Jiang Y, Liang X, Wang W, Chen C, Yuan Q, Zhang X, et al. Noninvasive Prediction of Occult Peritoneal Metastasis in Gastric Cancer Using Deep 
Learning. JAMA Netw Open (2021) 4:e2032269. doi: 10.1001/jamanetworkopen. 2020.32269

14. Huang Z, Liu D, Chen X, He D, Yu P, Liu B, et al. Deep Convolutional Neural Network Based on Computed Tomography Images for the Preoperative Diagnosis of Occult Peritoneal Metastasis in Advanced Gastric Cancer. Front Oncol (2020) 10:601869. doi: 10.3389/fonc.2020.601869

15. Giganti F, Antunes S, Salerno A, Ambrosi A, Marra P, Nicoletti R, et al. Gastric Cancer: Texture Analysis From Multidetector Computed Tomography as a Potential Preoperative Prognostic Biomarker. Eur Radiol (2017) 27:1831-9. doi: 10.1007/s00330-016-4540-y

16. Giganti F, Marra P, Ambrosi A, Salerno A, Antunes S, Chiari D, et al. PreTreatment MDCT-Based Texture Analysis for Therapy Response Prediction in Gastric Cancer: Comparison With Tumour Regression Grade at Final Histology. Eur J Radiol (2017) 90:129-37. doi: 10.1016/j.ejrad.2017.02.043

17. Liu S, Liu S, Ji C, Zheng H, Pan X, Zhang Y, et al. Application of CT Texture Analysis in Predicting Histopathological Characteristics of Gastric Cancers. Eur Radiol (2017) 27:4951-9. doi: 10.1007/s00330-017-4881-1

18. Liu S, Shi H, Ji C, Zheng H, Pan X, Guan W, et al. Preoperative CT Texture Analysis of Gastric Cancer: Correlations With Postoperative TNM Staging. Clin Radiol (2018) 73:756.e1-9. doi: 10.1016/j.crad.2018.03.005

19. Ma Z, Fang M, Huang Y, He L, Chen X, Liang C, et al. CT-Based Radiomics Signature for Differentiating Borrmann Type IV Gastric Cancer From Primary Gastric Lymphoma. Eur J Radiol (2017) 91:142-7. doi: 10.1016/ j.ejrad.2017.04.007

20. Hou Z, Yang Y, Li S, Yan J, Ren W, Liu J, et al. Radiomic Analysis Using Contrast-Enhanced CT: Predict Treatment Response to Pulsed Low Dose Rate Radiotherapy in Gastric Carcinoma With Abdominal Cavity Metastasis. Quant Imaging Med Surg (2018) 8:410-20. doi: 10.21037/qims.2018.05.01

21. Liu S, He J, Liu S, Ji C, Guan W, Chen L, et al. Radiomics Analysis Using Contrast-Enhanced CT for Preoperative Prediction of Occult Peritoneal Metastasis in Advanced Gastric Cancer. Eur Radiol (2020) 30:239-46. doi: 10.1007/s00330-019-06368-5

22. Kim HY, Kim YH, Yun G, Chang W, Lee YJ, Kim B. Could Texture Features From Preoperative CT Image be Used for Predicting Occult Peritoneal Carcinomatosis in Patients With Advanced Gastric Cancer. PloS One (2018) 13:e0194755. doi: 10.1371/journal.pone.0194755

23. Dong D, Tang L, Li ZY, Fang MJ, Gao JB, Shan XH, et al. Development and Validation of an Individualized Nomogram to Identify Occult Peritoneal Metastasis in Patients With Advanced Gastric Cancer. Ann Oncol (2019) 30:431-8. doi: 10.1093/annonc/mdz001

24. Wang S, Wang Q, Shao Y, Qu L, Lian C, Lian J, et al. Iterative Label Denoising Network: Segmenting Male Pelvic Organs in CT From 3d Bounding Box Annotations. IEEE Trans BioMed Eng (2020) 67:2710-20. doi: 10.1109/ TBME.2020.2969608

25. Saha BN, Ray N, Greiner R, Murtha A, Zhang H. Quick Detection of Brain Tumors and Edemas: A Bounding Box Method Using Symmetry. Comput Med Imaging Graph (2012) 36:95-107. doi: 10.1016/j.compmedimag.2011.06.001

26. Zhou J, Zhang Y, Chang KT, Lee KE, Wang O, Li J, et al. Diagnosis of Benign and Malignant Breast Lesions on DCE-MRI by Using Radiomics and Deep Learning With Consideration of Peritumor Tissue. J Magn Reson Imaging (2020) 51:798-809. doi: 10.1002/jmri.26981

27. Chang PD, Wong TT, Rasiej MJ. Deep Learning for Detection of Complete Anterior Cruciate Ligament Tear. J Digit Imaging (2019) 32:980-6. doi: 10.1007/s10278-019-00193-4

28. Jinnai S, Yamazaki N, Hirano Y, Sugawara Y, Ohe Y, Hamamoto R. The Development of a Skin Cancer Classification System for Pigmented Skin Lesions Using Deep Learning. Biomolecules (2020) 10. doi: 10.3390/ biom10081123

29. Wang XX, Ding Y, Wang SW, Dong D, Li HL, Chen J, et al. Intratumoral and Peritumoral Radiomics Analysis for Preoperative Lauren Classification in Gastric Cancer. Cancer Imaging (2020) 20:83. doi: 10.1186/s40644-020-00358-3

30. Hu Y, Xie C, Yang H, Ho J, Wen J, Han L, et al. Assessment of Intratumoral and Peritumoral Computed Tomography Radiomics for Predicting Pathological Complete Response to Neoadjuvant Chemoradiation in Patients With Esophageal Squamous Cell Carcinoma. JAMA Netw Open (2020) 3:e2015927. doi: 10.1001/jamanetworkopen.2020.15927

31. Li J, Zhang C, Wei J, Zheng P, Zhang H, Xie Y, et al. Intratumoral and Peritumoral Radiomics of Contrast-Enhanced CT for Prediction of Disease-
Free Survival and Chemotherapy Response in Stage II/III Gastric Cancer. Front Oncol (2020) 10:552270. doi: 10.3389/fonc.2020.552270

32. Jiang Y, Wang H, Wu J, Chen C, Yuan Q, Huang W, et al. Noninvasive Imaging Evaluation of Tumor Immune Microenvironment to Predict Outcomes in Gastric Cancer. Ann Oncol (2020) 31:760-8. doi: 10.1016/ j.annonc.2020.03.295

33. van Griethuysen J, Fedorov A, Parmar C, Hosny A, Aucoin N, Narayan V, et al. Computational Radiomics System to Decode the Radiographic Phenotype. Cancer Res (2017) 77:e104-104e107. doi: 10.1158/0008-5472.CAN-17-0339

34. Zwanenburg A, Vallières M, Abdalah MA, Aerts H, Andrearczyk V, Apte A, et al. The Image Biomarker Standardization Initiative: Standardized Quantitative Radiomics for High-Throughput Image-Based Phenotyping. Radiology (2020) 295:328-38. doi: 10.1148/radiol.2020191145

35. Jackson SH, Jamieson MJ, Johnston A, Shepherd AM. The Analysis of DoseResponse Curves-a Practical Approach. Br J Clin Pharmacol (1987) 23:199205. doi: 10.1111/j.1365-2125.1987.tb03030.x

36. Hanley JA, McNeil BJ. The Meaning and Use of the Area Under a Receiver Operating Characteristic (ROC) Curve. Radiology (1982) 143:29-36. doi: 10.1148/radiology.143.1.7063747

37. DeLong ER, DeLong DM, Clarke-Pearson DL. Comparing the Areas Under Two or More Correlated Receiver Operating Characteristic Curves: A Nonparametric Approach. Biometrics (1988) 44:837-45.

38. Wu J, Sun X, Wang J, Cui Y, Kato F, Shirato H, et al. Identifying Relations Between Imaging Phenotypes and Molecular Subtypes of Breast Cancer: Model Discovery and External Validation. J Magn Reson Imaging (2017) 46:1017-27. doi: 10.1002/jmri.25661

39. Braman NM, Etesami M, Prasanna P, Dubchuk C, Gilmore H, Tiwari P, et al. Intratumoral and Peritumoral Radiomics for the Pretreatment Prediction of Pathological Complete Response to Neoadjuvant Chemotherapy Based on Breast DCE-MRI. Breast Cancer Res (2017) 19:57. doi: 10.1186/s13058-017-0846-1

40. Wang X, Zhao X, Li Q, Xia W, Peng Z, Zhang R, et al. Can Peritumoral Radiomics Increase the Efficiency of the Prediction for Lymph Node Metastasis in Clinical Stage T1 Lung Adenocarcinoma on CT. Eur Radiol (2019) 29:6049-58. doi: 10.1007/s00330-019-06084-0

41. Lee AK, DeLellis RA, Silverman ML, Heatley GJ, Wolfe HJ. Prognostic Significance of Peritumoral Lymphatic and Blood Vessel Invasion in NodeNegative Carcinoma of the Breast. J Clin Oncol (1990) 8:1457-65. doi: 10.1200/JCO.1990.8.9.1457

42. Mohammed ZM, McMillan DC, Edwards J, Mallon E, Doughty JC, Orange C, et al. The Relationship Between Lymphovascular Invasion and Angiogenesis, Hormone Receptors, Cell Proliferation and Survival in Patients With Primary Operable Invasive Ductal Breast Cancer. BMC Clin Pathol (2013) 13:31. doi: $10.1186 / 1472-6890-13-31$

43. Pak KH, Jo A, Choi HJ, Choi Y, Kim H, Cheong JH. The Different Role of Intratumoral and Peritumoral Lymphangiogenesis in Gastric Cancer Progression and Prognosis. BMC Cancer (2015) 15:498. doi: 10.1186/ s12885-015-1501-9

44. Ikoma N, Blum M, Chiang YJ, Estrella JS, Roy-Chowdhuri S, Fournier K, et al. Yield of Staging Laparoscopy and Lavage Cytology for Radiologically Occult Peritoneal Carcinomatosis of Gastric Cancer. Ann Surg Oncol (2016) 23:43327. doi: $10.1245 / \mathrm{s} 10434-016-5409-7$

45. Ramos RF, Scalon FM, Scalon MM, Dias DI. Staging Laparoscopy in Gastric Cancer to Detect Peritoneal Metastases: A Systematic Review and MetaAnalysis. Eur J Surg Oncol (2016) 42:1315-21. doi: 10.1016/j.ejso.2016.06.401

46. Wang FH, Shen L, Li J, Zhou ZW, Liang H, Zhang XT, et al. The Chinese Society of Clinical Oncology (CSCO): Clinical Guidelines for the Diagnosis and Treatment of Gastric Cancer. Cancer Commun (Lond) (2019) 39:10. doi: 10.1186/s40880-019-0349-9

Conflict of Interest: Authors PY and HY were employed by company Infervision.

The remaining authors declare that the research was conducted in the absence of any commercial or financial relationships that could be construed as a potential conflict of interest.

Publisher's Note: All claims expressed in this article are solely those of the authors and do not necessarily represent those of their affiliated organizations, or those of the publisher, the editors and the reviewers. Any product that may be evaluated in 
this article, or claim that may be made by its manufacturer, is not guaranteed or endorsed by the publisher.

Copyright $\odot 2021$ Liu, Zhang, Hu, Yu, Zhang, Yin, Yang, Fang, Song, Wu, Hu and Huang. This is an open-access article distributed under the terms of the Creative
Commons Attribution License (CC BY). The use, distribution or reproduction in other forums is permitted, provided the original author(s) and the copyright owner(s) are credited and that the original publication in this journal is cited, in accordance with accepted academic practice. No use, distribution or reproduction is permitted which does not comply with these terms. 